\title{
A Micropower Cochlear Prosthesis System Demonstrator
}

\author{
Julius Georgiou $^{+}$, Timothy Constandinou ${ }^{+*}$ and Christopher Toumazou* \\ + Dept of Electrical and Computer Engineering, University of Cyprus, Nicosia, Cyprus \\ * Institute of Biomedical Engineering, Imperial College, London, UK \\ julio@ucy.ac.cy, t.constandinou@ucy.ac.cy, c.toumazou@imperial.ac.uk
}

Abstract - This demonstrator presents an ultra low power mixed-signal implementation of a cochlear prosthesis system. A laptop PC provides an audio stimulus to the cochlear chip. A microcontroller on the demonstrator board monitors the output channels of the chip and returns these to the $\mathrm{PC}$ to be displayed as a spectrogram. The audiological settings of the implant chip are controlled via a GUI running on the PC.

For more details on the cochlear chip please see:

J. Georgiou and C. Toumazou, "A Micropower Cochlear Prosthesis System," Circuits and Systems, 2003. ISCAS '03. Proc. of the 2003 IEEE Int. Symp. Vol. 3, pp. 834-837

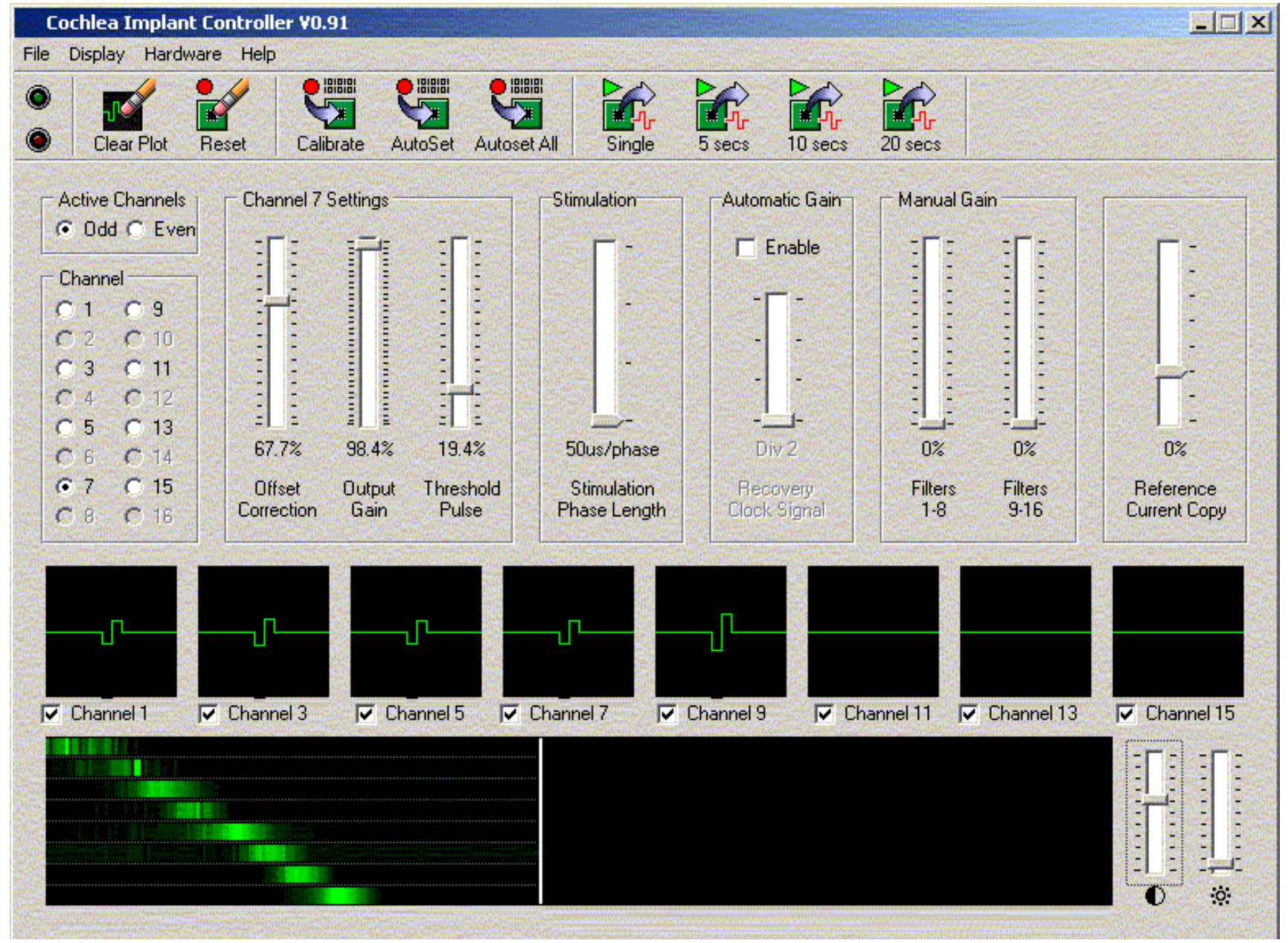

